\title{
Long non-coding RNA MEG3 alters neuronal apoptosis and is associated with poor prognosis in ischemic stroke: based on basic and prospective studies
}

\section{Meiping Wang}

Zhejiang Provincial People's Hospital

\section{Wenjuan Chen}

Zhejiang Provincial People's Hospital

\section{Yu Geng}

Zhejiang Provincial People's Hospital

\section{Chenghua $\mathrm{Xu}$}

Huangyan Hospital of Wenzhou Medical University

Xiaoxiao Tao

Huangyan Hospital of Wenzhou Medical University

Yi Zhang ( $D$ yi_zhang520@126.com)

Huangyan Hospital of Wenzhou Medical University

\section{Research article}

Keywords: Ischemic stroke, Prognosis, Long non-coding RNA, Maternally expressed gene 3

Posted Date: June 5th, 2019

DOI: https://doi.org/10.21203/rs.2.10093/v1

License: (c) This work is licensed under a Creative Commons Attribution 4.0 International License.

Read Full License 


\section{Abstract}

Background: Ischemic stroke (IS) is a highly heterogeneous disease with variable pathogenesis. Due to the lack of early predictive markers, the mortality rate of IS remains high worldwide. This study focused on the expression pattern of long non-coding RNA (IncRNA) maternally expressed gene 3 (MEG3) and its prognostic value in IS. Methods: Twenty-four ICR mice (30-35g, 12 males and 12 females) were involved in this study. Middle cerebral artery occlusion (MCAO) was established to simulate an IS environment. Oxygen-glucose deprivation/reoxygenation (OGD/R) in cells were also performed. A total of 215 IS patients and 153 age and gender-matched controls were included. Quantitative Real-time PCR (qRT-PCR) was performed to detect the expression of MEG3 in peripheral blood leukocytes. Results: The MEG3 expression in MCAO group was significantly higher than control group $(P=0.004)$, and the survival time of high MEG3 group was significantly lower than that of the low MEG3 group ( $P=0.042)$. OGD/R promotes cell apoptosis, while si-MEG3 inhibits apoptosis. Results of WB showed that, expression level of Bax was down regulated by si-MEG3, while OGD/R increased its expression level. MEG3 was significantly upregulated in IS patients and associated with the National Institutes of Health Stroke Scale (NIHSS) $(r=0.347, P<0.001)$, modified Rankin Scale ( $m R S)(r=0.385, P<0.001)$, high sensitivity C-reactive protein (Hs-CRP) $(r=0.221, P=0.002)$ level and infarct volume $(r=0.201, P=0.006)$. Overall survival analysis showed that patients with higher MEG3 expression had a relatively poor prognosis $(P<0.001)$. Meanwhile, multivariate analysis revealed that MEG3 was an independent prognostic marker of functional outcome and death in patients with IS. Conclusions: Current study suggested that MEG3 might be considered as a potential prognostic indicator in IS.

\section{Background}

Ischemic stroke (IS) is the most common type of stroke, accounting for $60 \%-70 \%$ of all strokes [1]. The mortality rate of IS in China is about $10 \%$, the recurrence rate of survivors is about $40 \%$, and the disability rate is more than $50 \%$ [1]. IS diagnosis is based primarily on imaging and neurological impairment. Unfortunately, current intervention and treatment of IS is still limited, including the following aspects: (1) Ischemic damage in brain tissue is not obvious within 24 hours after ischemic stroke by CT, and CT is mainly used to quickly eliminate intracranial tumors or cerebral hemorrhage [2]. (2) MRI has higher sensitivity and specificity than CT, but most medical institutions in China do not have the ability to conduct MRI examinations [2]. (3) A recent systematic review suggested that the survival rate of IS patients who can receive thrombolytic therapy within 6 hours of symptom onset can be increased by $46.0 \%$, and the disability rate caused by IS can be reduced by $42.1 \%$ [3]. Unfortunately, only about $2 \%$ of patients are able to receive treatment in a timely manner [3]. An easily-accessible test with estimate of the severity of disease and prognosis is pivotal for optimized care and allocation of health care resources to improve the outcome.

Increasing evidence has pointed to a relationship between long non-coding RNA (IncRNA) and cardiovascular disease, including atherosclerosis-related smooth muscle cell (SMC) [4], endothelial cell (EC) [ 5] and lipid metabolism regulation [ 6]. LncRNA maternally expressed gene 3 (MEG3) is a newly 
discovered non-coding RNA lying on the strongest genetic susceptibility locus for cardiovascular disease in the chromosome 14q9 [7]. Previous studies showed that MEG3 is highly expressed in endothelial, smooth muscle and immune cells and may be involved in the atherosclerotic process such as in plaque stability [7]. However, there are little reports about the basic and clinical evaluation of the prognostic value of MEG3 in IS.

In the present study, oxygen-glucose deprivation/reoxygenation (OGD/R) in cells and middle cerebral artery occlusion (MCAO) in mice were performed to simulate an IS environment. MEG3 expression and its prognostic value in IS patients was also investigated. Then, the relationship between MEG3 expression and clinical characteristics was also analyzed. The purpose of this study was to explore molecular marker for prognosis assessment of IS.

\section{Methods}

\subsection{MCAO model establishment}

All animals were treated according to the National Institute of Health Guidelines for the Care and Use of Laboratory Animals. All experimental procedures were approved by the Animal Care and Use Committee of the Zhejiang University (authorization No: 201804011). In the process of feeding and experimenting with animals, humane care was given according to the $3 \mathrm{R}$ principle.

Twenty-four ICR mice (30-35g, mean weight was 32g, 12 males and 12 females) were purchased from the Experimental Animal Center of Zhejiang University School of Medicine [SCXK (Z) 2017-0008]. Twentyfour mice were randomly divided into MCAO group $(n=12)$ and control group $(n=12)$. MCAO group was intraperitoneally injected with $10 \mathrm{mg} / \mathrm{kg}$ xylazine. The left middle cerebral artery was permanently blocked by a single-wire nylon suture through the external carotid artery into the internal carotid artery to the origin of middle cerebral artery. The control group used the same surgery except for the insertion of sutures. Mouse cerebral blood flow was detected by B-ultrasound, and the reduction of hemi-cerebral blood flow confirmed the successful MCAO modeling. At the time of modeling and $1 \mathrm{~h}$ after successful modeling, $40 \mu \mathrm{L}$ tail vein blood of each mouse was collected. Subsequently, both groups of mice were observed for 8 weeks.

After 8 weeks of follow-up observation, the animals (control group, $n=12$; MCAO group, $n=12$ ) were deeply anesthetized by an overdose of chloral hydrate $(400 \mathrm{mg} / \mathrm{kg})$, the brains were removed and placed in formalin solution until slicing.

\subsection{Cell culture}

Human brain microvascular endothelial cells (HBMECs) obtained from the Center for Excellence in Brain Science and Intelligence Technology, Chinese Academy of Sciences Institute of Neuroscience, Chinese 
Academy of Sciences (Shanghai, China) were plated in 96 -well plates at a density of $5 \times 10^{4}$ cells/well and cultured in high-glucose Dulbecco's Modified Eagle Medium (DMEM; Gibco, Grand Island, NY, USA, 4.5 g/L glucose) supplemented with $4 \mathrm{mM}$ glutamine, sodium pyruvate (Sigma, St. Louis, MO, USA) and 10\% fetal bovine serum (Gibco, Rockville, MD, USA). The cells grew in a $37^{\circ} \mathrm{C}$ incubator with $5 \% \mathrm{CO}_{2}$.

\subsection{Cell transfection}

MEG3 and negative control (NC) siRNA were purchased from Genepharm. Co., (Shanghai, China). The transfections were performed using Lipofectamine 2000 (Invitrogen, NY, USA) according to the manufactures' instructions. Cells were cultured, and level of MEG3 was examined $48 \mathrm{~h}$ post-transfection.

\subsection{Oxygen glucose deprivation/reoxygenation (OGD/R)}

Cells were treated with OGD/R exposure as previously described [8]. Briefly, balanced salt solution was adopted to wash cells twice, which were then subjected to OGD medium without glucose. Later, cells were placed in an anaerobic chamber containing $5 \% \mathrm{CO}_{2}$ at $37^{\circ} \mathrm{C}$ for $2 \mathrm{~h}$. After adding $5 \mathrm{mM}$ glucose to the medium, cells were returned to full culture medium and normoxia for $24 \mathrm{~h}$. Control cells were cultured in a regular $5 \% \mathrm{CO}_{2}$ incubator containing $5 \mathrm{mM}$ glucose at $37^{\circ} \mathrm{C}$ in the same buffer.

\subsection{Western blot analysis}

Cells were lysed using RIPA Lysis Buffer (Beyotime, China). Equal amounts of proteins ( $30 \mathrm{mg}$ ) were separated by $10 \%$ SDS-PAGE gels and then transferred to a polyvinyl difluoride (PVDF) membrane. After soaked in Protein Free Rapid Block Buffer (Beyotime, China), the membrane was incubated overnight at $4^{\circ} \mathrm{C}$ with one of the following antibodies: rabbitmonoclonal Bax, and rabbit monoclonal $\beta$-catenin (1:500; Affinity, USA). Then the membrane was incubated with a secondary anti-rabbit antibody (1:4000; Affinity, USA) for $1 \mathrm{~h}$ at room temperature. Finally, the membrane was visualized by ECL-PLU (AmershamBiosciences.Sweden).

\subsection{Flow cytometry}

Apoptosis was evaluated with Annexin V fluorescein isothiocyanate (FITC) / apoptosis detection kit (Sigma, San Francisco, CA, USA). Cell suspensions were exposed to $5 \mu \mathrm{l}$ annexin V-FITC and $10 \mu \mathrm{l}$ propidium iodide for $15 \mathrm{~min}$ at $15-25^{\circ} \mathrm{C}$ Stained cells were carefully analyzed using a BD FACS Calibur ${ }^{\mathrm{TM}}$ flow cytometer (BD Biosciences, Piscataway, NJ, USA). 


\subsection{Experimental subjects}

We recruited 215 patients (138 men and 77 women; median age: 73, interquartile range [IQR]: 64-81) who were diagnosed as IS between October 2016 and May 2018 at Zhejiang Provincial People's Hospital, Hangzhou, China. The National Institutes of Health Stroke Scale (NIHSS) score was assessed [9]. IS was classified into three groups: large-artery atherosclerosis (LAA), cardioembolism (CE) and small vessel occlusion (SVO). MRI with diffusion-weighted imaging (DWI) was performed in some patients. We also collected 153 age and gender-matched controls (101 men and 52 women; median age: 71, IQR: 65-76) from physical examination center. Blood samples of IS were collected after the admission (within 0-12 [ $n=64], 12-24$ [ $n=108], 24-36$ [ $n=32], 36-48$ [ $n=11]$ hours from symptom onset). Blood samples of controls were collected at the time of physical examination.

\subsection{Ethics approve and consent to participate}

This study was all approved by the Ethics Committee of Zhejiang Provincial People's Hospital (Hangzhou, China). Written informed consent was provided in accordance with the Declaration of Helsinki.

\subsection{RNA isolation, cDNA synthesis and Quantitative Real-time PCR (qRT-PCR)}

Expression level of MEG3 was performed on the Bio-Rad CFX96 (Inc., Hercules, CA, USA) using SYBR Green qPCR Mix according to manufacturer's procedures. The expression of MEG3 was normalized to Glyceraldehyde-3-Phosphate Dehydrogenase (GAPDH). All the experiments were in triplicates. MEG3 level was calculated using $2^{-\Delta \mathrm{Ct}}$ method.

\subsection{Follow-Up and End Points}

Prognosis of patients were obtained on 6 months after hospitalization according to modified Rankin Scale (mRS) [10] blinded to MEG3 level. The primary end point was favorable functional outcome after 6 months. Secondary end point was death within 6 months follow-up.

\subsection{Statistical analysis}

Statistical analyses were performed using SPSS version 19.0. All data were presented as mean \pm standard deviation $(\mathrm{M} \pm \mathrm{SD}$ ) or median (interquartile range, IQR) or rate (\%). The differences between normally distributed numeric variables were evaluated by Student's t-test, whereas non-normally distributed variables were analyzed by Mann-Whitney U-test. Categorical variables were analyzed by $\chi^{2}$ 
test. Correlations were analyzed using Spearman correlation. Association between IS and levels of MEG3 was calculated through binary logistic regression analysis. Survival curve was calculated by KaplanMeier method. $P<0.05$ was considered to be statistically significant.

\section{Results}

\subsection{MEG3 expression was up-regulated in MCAO model mice}

Figure 1A provides images of mouse brain sections in response to MCAO. After hypoxia-induced ischemic infarct in mice, tail vein blood was collected and analyzed. The production of MEG3 was detected using qRT-PCR method. In this study, we found that the MEG3 expression in MCAO group was significantly higher than control group ( $P=0.004$, Figure 1B). Besides, in the MCAO group, the survival time of the high MEG3 group was significantly lower than that of the low MEG3 group (Log-rank test $P=0.042$, Figure $1 C$ ).

\section{2. si-MEG3 inhibits neuronal apoptosis}

Apoptosis of HBMECs in response to various disturbances is clearly displayed in Figure 2A as assessed by flow cytometry. Results suggest that OGD/R promotes apoptosis, while si-MEG3 inhibits apoptosis. Results of WB showed that, expression level of Bax was down regulated by si-MEG3, while OGD/R increased its expression level (Figure 2B).

\subsection{MEG3 expression in peripheral blood leukocytes was significantly up-regulated in IS patients}

In current study, 215 patients completed 6 months follow-up. The main demographic and clinical characteristics of subjects were summarized in Table 1. No difference was observed in regard to important risk factors including age, gender, body mass index (BMI), smoking, diabetes, TG, LDL-C and $\mathrm{Lp}(\mathrm{a})$ between IS patients and the controls. However, there was a significant difference in hypertension, hypercholesterolaemia, alcoholism, HDL-C, TC, leucocyte count and Hs-CRP between IS patients and the controls $(P<0.05)$.

To observe the value of MEG3 as biomarker, MEG3 expression was measured by qRT-PCR. Present study showed that MEG3 expression relative to GAPDH in IS patients was significantly up-regulated than in controls $(P<0.001$, Figure 3A). Further research indicated that MEG3 expression in LAA group was higher than that in CE, SVO and the controls (LAA vs CE, $P=0.003$; LAA vs $S V O, P<0.001$; LAA vs the controls, $P<0.001$, Figure 3B), and MEG3 level in CE and SVO was significantly higher than that in the controls (CE 
vs the controls, $P<0.001$; SVO vs the controls, $P<0.001$, Fig. 3B). However, upon comparison of the level in CE and SVO group, no marked difference was found. Of note, when MEG3 expression was divided into quartiles, we also found that the proportion of LAA strokes increased according to the elevated level of MEG3 $(P<0.001$, Table 2).

In univariate analysis, increasing MEG3 ( $P<0.001, \mathrm{OR}=1.409,95 \% \mathrm{Cl}: 1.144-1.675)$ level showed significant association with IS. After adjusting for relevant clinical and laboratory variables, elevated MEG3 $(P<0.001$, adjusted $\mathrm{OR}=1.268,95 \% \mathrm{Cl}$ : $1.088-1.450)$ level remained significant with regard to increased odds of having IS.

\subsection{Correlation between MEG3 expression and clinical variables}

We detected the correlation between MEG3 expression and clinical parameters in the 215 IS patients. As shown in Figure 4, the MEG3 expression was significantly correlated with NIHSS scores $(r=0.347$, $P<0.001$, Figure 4A), mRS ( $r=0.385, P<0.001$, Figure 4C) and Hs-CRP $(r=0.221, P=0.002$, Figure 4D). Moreover, there was a significant positive association between MEG3 level and infarct volume $(r=0.201$, $P=0.006$, Figure 4B).

\subsection{MEG3 expression and 6-Month Functional Outcome}

In our study, an unfavorable functional outcome was found in 84 patients (39.1\%) with a median mRS score of 4 (IQR 3-5). In the 84 patients with an unfavorable functional outcome, MEG3 level was higher compared with those in patients with a favorable outcome (Figure 5A). In univariate logistic regression analysis, MEG3 level as compared with Hs-CRP, the NIHSS score and other risk factors was presented in Table 3. With an unadjusted OR of 2.504 (95\% Cl: 1.147-5.466, P<0.001), MEG3 had a strong association with unfavorable functional outcome. After adjusting for all other significant outcome predictors, MEG3 remained an independent unfavorable outcome predictor with an adjusted OR of 1.998 (95\% Cl 1.144-3.491, P<0.001). In addition, the NIHSS score and Hs-CRP remained significant outcome predictors, unlike all others assessed (Table 3 ).

\subsection{MEG3 expression and 6-Month Mortality}

At 6 months, 42 patients (19.5\%) had died. Nonsurvivors had significantly higher MEG3 level than survivors (Figure 5B). In univariate logistic regression analysis, MEG3 level as compared with Hs-CRP, the NIHSS score and other risk factors was presented in Table 3. With an unadjusted OR of $1.638(95 \% \mathrm{Cl}$ 1.202-2.232, $P=0.002)$, MEG3 level was an independent predictor for mortality. After adjusting for all other 
significant outcome predictors, MEG3 remained an independent mortality predictor with an adjusted OR of $1.512(95 \% \mathrm{Cl} 1.058-2.159, P=0.023)$. In addition, the NIHSS score and Hs-CRP remained significant outcome predictors, unlike all others assessed (Table 3 ).

To answer whether MEG3 expression correlated to the outcome of IS patients, the 215 IS patients were divided into two groups: relative high MEG3 group ( $n=108$, MEG3 expression $\geq$ median) and relative low MEG3 group ( $n=107$, MEG3 expression < median) according to the median expression of MEG3. Time to death was calculated by the Kaplan-Meier method and log-rank test. Significantly, patients in the highMEG3 group had a higher risk of death compared with low-MEG3 group (Log-rank test $P<0.001$, Figure 6 ).

\section{Discussion}

LncRNA has drawn considerable attention for its effects in various diseases, including neurological disorders. In the present study, for the first time, we investigated the clinical value of MEG3 in MCAO model mice and IS patients. We found that the level of MEG3 in MCAO group was significantly higher than the control group, and the survival time of high MEG3 group was significantly lower than that of the low MEG3 group. Results also suggested that OGD/R promoted apoptosis, while si-MEG3 inhibited apoptosis. Moreover, we also found that the MEG3 level in peripheral blood leukocytes was significantly higher in IS patients after adjustment for relevant covariates. When further considering the subtypes of IS, we documented that MEG3 had differential association patterns among stroke subtypes. LAA stroke was associated with highest expression of MEG3, and MEG3 level in CE and SVO was also significantly higher than that in the controls. However, upon comparison of the levels in the CE and SVO group, no marked difference was found. Considering the role of atherosclerotic plaques in the development of LAA stroke and their association with MEG3, we may suppose that MEG3 is an important participant in early atherosclerosis, consequently, IS. Meanwhile, our data showed that MEG3 expression was related to infarct volume and stroke severity. In addition, comparing with the low MEG3 group, the increased levels of MEG3 were associated with a poor prognosis and a shorter survival time.

It is generally accepted that genetic variants on chromosome $14 q 9$ are associated with the risk of coronary artery disease. Of note, MEG3 expression has been elaborated to be affected by several polymorphisms in the $14 q 9$ locus, and this differential expression may regulate the expression levels of genes involved in several atherogenic pathways, in turn, finally impact in the risk of cardiovascular disease [11]. An explanation interpretation of the expression profiling results is the role of MEG3 in coordinating vascular remodeling. MEG3 depletion caused the upregulation of two important antiapoptotic proteins, bcl-2-related protein A1 (BCL2A1) and baculoviral IAP repeat containing 3 (BIRC3) [ 12]. VSMC apoptosis can perform major changes in arterial architecture, especially when coordinated with matrix turnover and VSMC proliferation. These two cellular activities strongly regulated by matrix metalloproteinase-3 (MMP-3) and Heparin binding EGF like growth factor (HBEGF), which were also affected by MEG3 [11]. Moreover, Holdt et al. [13] also found that MEG3 may be involved in the atherosclerotic process such as in vascular remodeling, thrombogenesis, and plaque stability. Previous studies have demonstrated that MEG3 may play an important role in cardiovascular disease. Therefore, 
we supposed that MEG3 might be involved in IS. These studies indicated that MEG3 could participate in the development of IS as well as other cardiovascular disease. Unfortunately, the exact mechanism of MEG3 is still unknown.

This study has several limitations. First, the sample size is relatively small, therefore, present findings should be validated in trials with more cases in future. Second, this study was conducted in a single hospital, thereby being prone to selection bias. Multi-center research is still needed to further validate our conclusions. Third, current study did not explore the potential mechanism of MEG3 affecting the prognosis of IS. In the future, corresponding cell and animal experiments can be designed for further exploration.

\section{Conclusions}

Present study elaborated that the MEG3 expression was significantly higher in LAA strokes than in nonLAA strokes and controls. In addition, our study indicated that MEG3 was an independent prognostic factor of IS patients, and comparing with the high-MEG3 group, the low-MEG3 group had a good prognosis and a longer survival time. These findings suggested for the first time that peripheral blood leukocytes MEG3 could be used as a novel prognostic marker for IS. Future studies are warranted to test exact molecular mechanism and to assess the mechanism responsible for the unregulated expression of MEG3 in IS.

\section{List Of Abbreviations:}

IS, ischemic stroke; LncRNA, long non-coding RNA; MEG3, maternally expressed gene 3; MCAO, middle cerebral artery occlusion; qRT-PCR, quantitative Real-time PCR; NIHSS, National Institutes of Health Stroke Scale; mRS, modified Rankin Scale; Hs-CRP, high sensitivity C-reactive protein; ROC, receiver operating characteristic curve; LAA, large-artery atherosclerosis; CE, cardioembolism; SVO, small vessel occlusion; DWI, diffusion-weighted imaging; AUC, area under the ROC curve.

\section{Declaration}

Ethics approval and consent to participate: This study was all approved by the Ethics Committee of Zhejiang Provincial People's Hospital (Hangzhou, China). Written informed consent was provided in accordance with the Declaration of Helsinki. All animal experiments and surgical procedures were approved by the Experimental Animal Center of Zhejiang University School of Medicine (201804011).

Consent for publication: All authors have contributed to, read and approved the final manuscript for publication.

Availability of data and material: All data generated or analysed during this study are included in this published article. 
Competing interests: The authors declare that they have no competing interests.

Funding: YZ was supported by a grant from the Foundation of Zhejiang provincial science and Technology Department (No. 2016C40102).

Authors' contributions: YZ conceived the project and designed the experiments. MW, WC, YG, CX and XT conducted the experiments. MW and CX wrote the manuscript. WC, $Y G$ and $Y Z$ revised the manuscript.

Acknowledgments: We thank all of subjects enrolled into this study.

\section{References}

[1] Kim BJ, Lee SH, Ryu WS, et al. Adipocytokines and ischemic stroke: differential associations between stroke subtypes. J Neurol Sci. 2012;312(1-2):117-22.

[2] Zhu M, Zuo J, Shen J, et al. Diagnostic Potential of Differentially Expressed Homer1 and Homer2 in Ischemic Stroke. Cell Physiol Biochem. 2016;39(6):2353-63.

[3] Wardlaw JM, Murray V, Berge E, et al. Recombinant tissue plasminogen activator for acute ischaemic stroke: an updated systematic review and meta-analysis. Lancet. 2012;379(9834):2364-72.

[4] Motterle A, Pu X, Wood H, et al. Functional analyses of coronary artery disease associated variation on chromosome 9p21 in vascular smooth muscle cells. Hum Mol Genet. 2012;21(18):4021-9.

[5] Kurian L, Aguirre A, Sancho-Martinez I, et al. Identification of novel long noncoding RNAs underlying vertebrate cardiovascular development. Circulation. 2015;131(14):1278-90.

[6] Halley P, Kadakkuzha BM, Faghihi MA, et al. Regulation of the apolipoprotein gene cluster by a long noncoding RNA. Cell Rep. 2014;6(1):222-30.

[7] Bai Y, Zhang Q, Su Y, et al. Modulation of the Proliferation/Apoptosis Balance of Vascular Smooth Muscle Cells in Atherosclerosis by IncRNA-MEG3 via Regulation of miR-26a/Smad1 Axis. Int Heart J. 2019;60(2):444-50.

[8] Mo ZT, Fang YQ, He YP, et al Beta-Asarone protects PC12 cells against OGD/R-induced injury via attenuating Beclin-1-dependent autophagy. Acta Pharmacol Sin. 2012;33(6):737-42.

[9] Brott T, Marler JR, Olinger CP, et al. Measurements of acute cerebral infarction: lesion size by computed tomography. Stroke. 1989;20(7):871-5.

[10] Bonita R, Beaglehole R. Recovery of motor function after stroke. Stroke. 1988;19(12):1497-500.

[11] Huang P, Huang FZ, Liu HZ, et al. LncRNA MEG3 functions as a ceRNA in regulating hepatic lipogenesis by competitively binding to miR-21 with LRP6. Metabolism. 2019;94:1-8. 
[12] Thomadaki H, Scorilas A. BCL2 family of apoptosis-related genes: functions and clinical implications in cancer. Crit Rev Clin Lab Sci. 2006;43(1):1-67.

[13] Holdt LM, Sass K, Gäbel G, et al. Expression of Chr9p21 genes CDKN2B (p15(INK4b)), CDKN2A (p16(INK4a), p14(ARF)) and MTAP in human atherosclerotic plaque. Atherosclerosis. 2011;214(2):264-70.

\section{Tables}

Table 1. Characteristics of the studied subjects 
Characteristics

Patients $(n=215) \quad$ Normal cases $(n=$

$P$

153)

Age (years), median (IQR)

Male sex (\%)

$73(64,81)$

$71(65,76)$

$0.062^{\mathrm{a}}$

Male sex $(\%)$

64.2

66.0

$0.718^{b}$

BMI $\left(\mathrm{kg} / \mathrm{m}^{2}\right)(\mathrm{IQR})$

$23.8(22.4,25.7)$

$23.7(22.3,24.8)$

$0.279^{\mathrm{a}}$

Infarct volume $(\mathrm{mL}, \mathrm{IQR} ; \mathrm{n}=$

$22(11,32)$

186)

NIHSS at admission, median

$9(5,14)$

(IQR)

mRS at 6 month, no. (\%)

$0-2$

63.3

3-6

36.7

Vascular risk factors (\%)

Hypertension

65.1

48.4

$0.001^{\mathrm{b}}$

Diabetes mellitus

28.8

25.5

$0.479^{\mathrm{b}}$

Hypercholesterolaemia

29.3

14.4

$0.001^{b}$

Smoking

27.4

18.9

$0.060^{\mathrm{b}}$

Alcoholism

20.9

13.1

$0.041^{b}$

Laboratory findings median (IQR)

Total cholesterol (mg/dL)

$161.2(125.5$,

$153.8(136.2,172.7)$

$0.024^{\mathrm{a}}$ 206.3)

Triglycerides (mg/dL)

$125.8(96.1,154.2) \quad 111.1(82.3,193.9)$

$0.481^{\mathrm{a}}$

High-density lipoproteins

$43.2(33.8,51.1)$

$52.5(41.9,62.9)$ $<0.001^{\mathrm{a}}$ $(\mathrm{mg} / \mathrm{dL})$

Low-density lipoproteins (mg/dL)

Lipoprotein (a) (mg/L)

$101.2(72.8,127.2)$

$102.1(85.7,120.3)$

$0.622^{\mathrm{a}}$

$173.4(125.9$,

$167.3(105.1,302.7)$

$0.824^{\mathrm{a}}$ 
Leucocyte count $\left(\times 10^{9} / \mathrm{L}\right)$

Hs-CRP (mg/dL)

Stroke etiology (\%)

Large-artery arteriosclerosis

Small-artery occlusion

Cardioembolism
$9.2(7.4,11.1)$

$0.98(0.63,2.11)$
$8.0(6.7,9.3)$

$0.51(0.27,0.82)$ $<0.001^{\mathrm{a}}$

$<0.001^{\mathrm{a}}$
47.0

31.1

21.9

Data are percentage or median (25\% percentiles, $75 \%$ percentiles). Abbrebiations: ${ }^{\mathrm{a}}$ : MannWhitney U-test; ${ }^{\text {b}}$ : Chi-square test; IQR: interquartile range; BMI: body mass index; NIHSS: National Institutes of Health Stroke Scale; mRS: modified Rankin Scale; Hs-CRP: high sensitivity C-reactive protein; $\mathrm{P}<0.05$ was considered statistically significant.

Table 2. Participants' characteristics according to the quartiles of MEG3

\begin{tabular}{lccccc}
\hline \multirow{2}{*}{ Subject groups } & \multicolumn{4}{c}{ Quartiles of MEG3, range, relative expression (-log), N=368 } & \\
\cline { 2 - 5 } & 1 st, <1.722 & 2nd, 1.722-2.065 & 3rd, 2.065-2.455 & 4 th, $>2.455$ & $P$ \\
\hline LAA & $40(43.5 \%)$ & $33(35.9 \%)$ & $24(26.0 \%)$ & $4(4.3 \%)$ & $<0.001$ \\
CE & $11(12.0 \%)$ & $9(9.8 \%)$ & $11(12.0 \%)$ & $16(17.5 \%)$ & 0.458 \\
SVO & $14(15.2 \%)$ & $18(19.6 \%)$ & $15(16.3 \%)$ & $20(21.7 \%)$ & 0.648 \\
Control & $27(29.3 \%)$ & $32(34.7 \%)$ & $42(45.7 \%)$ & $52(56.5 \%)$ & 0.001 \\
\hline
\end{tabular}

Abbreviation: LAA: large-artery atherosclerosis; CE: cardioembolism; SVO: small vessel occlusion.

Table 3. Univariate and multivariate logistic regression analyses for outcome and mortality 


\begin{tabular}{|c|c|c|c|c|}
\hline \multirow[t]{2}{*}{ Parameter } & \multicolumn{2}{|l|}{ Univariate analysis } & \multicolumn{2}{|l|}{ Multivariate analysis } \\
\hline & OR $(95 \% \mathrm{CI})^{\mathrm{a}}$ & $P$ & OR $(95 \% \mathrm{CI})^{\mathrm{a}}$ & $P$ \\
\hline \multicolumn{5}{|l|}{ Predictor: functional outcome } \\
\hline Age & $1.001(0.970,1.032)$ & 0.995 & & \\
\hline Male sex & $1.450(0.658,3.193)$ & 0.356 & & \\
\hline BMI $\left(\mathrm{kg} / \mathrm{m}^{2}\right)$ & $1.060(0.923,1.217)$ & 0.410 & & \\
\hline NIHSS & $1.641(1.323,1.979)$ & 0.021 & $1.297(1.184,1.425)$ & 0.015 \\
\hline MEG3 $^{\mathrm{b}}$ & $2.504(1.147,5.466)$ & $<0.001$ & $1.998(1.144,3.491)$ & $<0.001$ \\
\hline Hypertension & $1.663(0.779,3.549)$ & 0.189 & & \\
\hline Hypercholesterolemia & $1.559(0.695,3.495)$ & 0.281 & & \\
\hline Diabetes mellitus & $0.786(0.518,1.380)$ & 0.077 & & \\
\hline Smoking & $1.121(0.473,2.655)$ & 0.796 & & \\
\hline Alcoholism & $0.766(0.306,1.915)$ & 0.568 & & \\
\hline Leucocyte count $\left(\times 10^{9} / \mathrm{L}\right)$ & $1.077(0.952,1.161)$ & 0.402 & & \\
\hline Hs-CRP (mg/dL) & $1.927(1.510,2.466)$ & $<0.001$ & $1.433(1.168,1.754)$ & 0.011 \\
\hline \multicolumn{5}{|l|}{ Predictor: death } \\
\hline Age & $0.999(0.962,1.038)$ & 0.972 & & \\
\hline Male sex & $0.788(0.401,1.534)$ & 0.480 & & \\
\hline BMI $\left(\mathrm{kg} / \mathrm{m}^{2}\right)$ & $1.029(0.875,1.211)$ & 0.728 & & \\
\hline NIHSS & $1.008(1.002,1.015)$ & 0.014 & $1.002(0.997,1.009)$ & 0.181 \\
\hline MEG3 $^{b}$ & $1.638(1.202,2.232)$ & 0.002 & $1.512(1.058,2.159)$ & 0.023 \\
\hline Hypertension & $1.023(0.995,1.035)$ & 0.152 & & \\
\hline Hypercholesterolemia & $1.236(0.467,3.272)$ & 0.670 & & \\
\hline Diabetes mellitus & $0.979(0.362,2.650)$ & 0.967 & & \\
\hline Smoking & $0.924(0.335,2.547)$ & 0.879 & & \\
\hline Alcoholism & $2.032(0.603,6.846)$ & 0.253 & & \\
\hline Leucocyte count $\left(\times 10^{9} / \mathrm{L}\right)$ & $1.056(0.951,1.174)$ & 0.450 & & \\
\hline Hs-CRP (mg/dL) & $1.261(1.061,1.499)$ & 0.009 & $1.276(1.040,1.567)$ & 0.020 \\
\hline
\end{tabular}


Abbreviation: OR: odds ratio; CI: confidence interval; BMI: body mass index; NIHSS: National Institutes of Health Stroke Scale; Hs-CRP: high sensitivity C-reactive protein. $P<0.05$ was considered statistically significant.

a Note that the odds ratio corresponds to a unit increase in the explanatory variable

$\mathrm{b}^{\mathrm{b}}$ Log-transformed to achieve normal distribution. Note that the odds ratio corresponds to a log unit increase in the explanatory variable

\section{Figures}

A

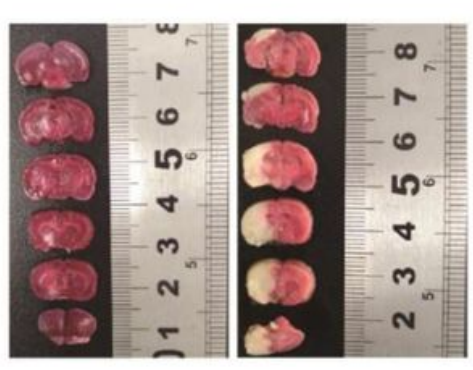

Control
B

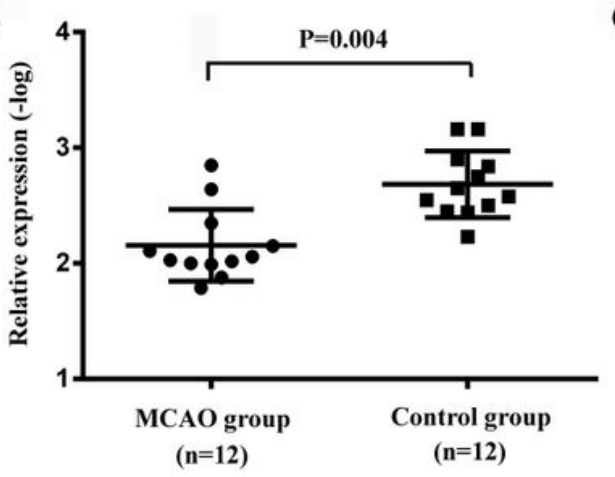

C

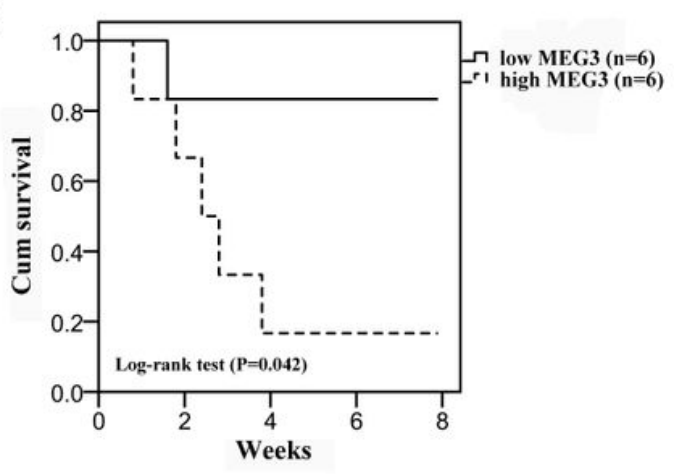

\section{Figure 1}

The expression level and prognostic value of IncRNA MEG3 in MCAO. (A). Images of mouse brain sections in response to MCAO. (B). MEG3 level in MCAO was significantly higher than that in controls. (C). In MCAO group, the survival time of the high MEG3 group was significantly lower than that of the low MEG3 group. 
A

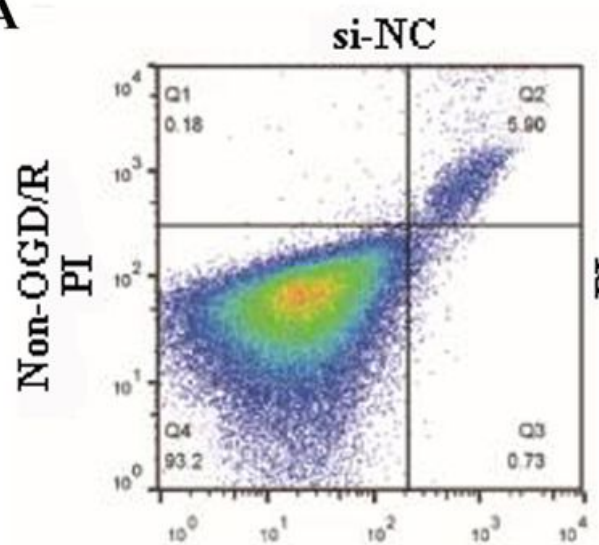

Annexin V-FITC

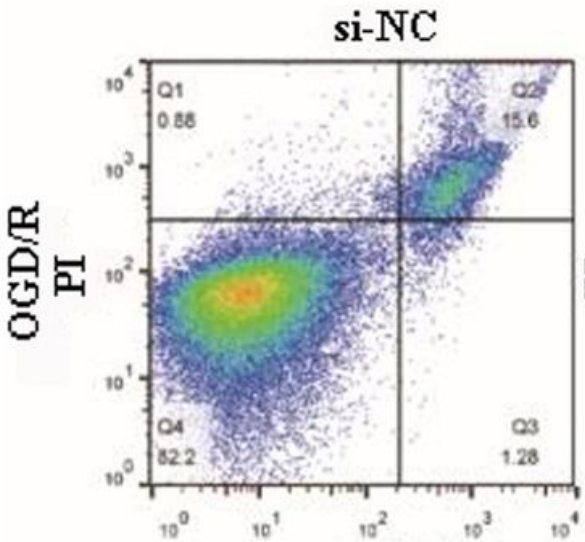

Annexin V-FITC

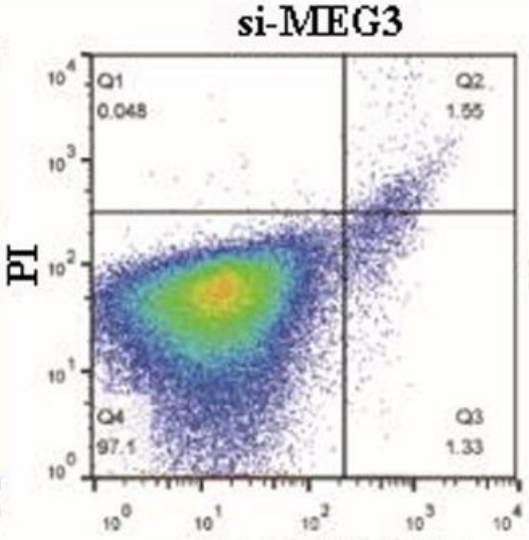

Annexin V-FITC

si-MEG3

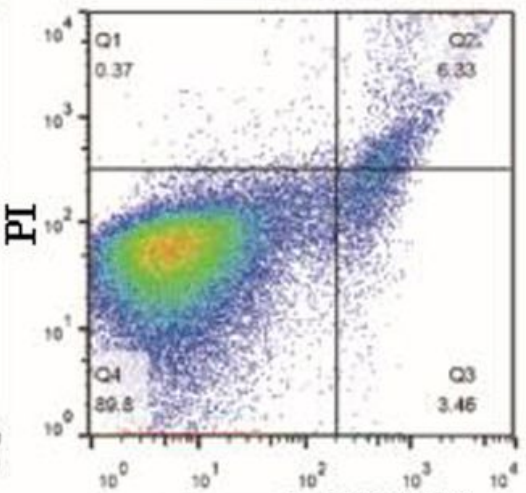

Annexin V-FITC
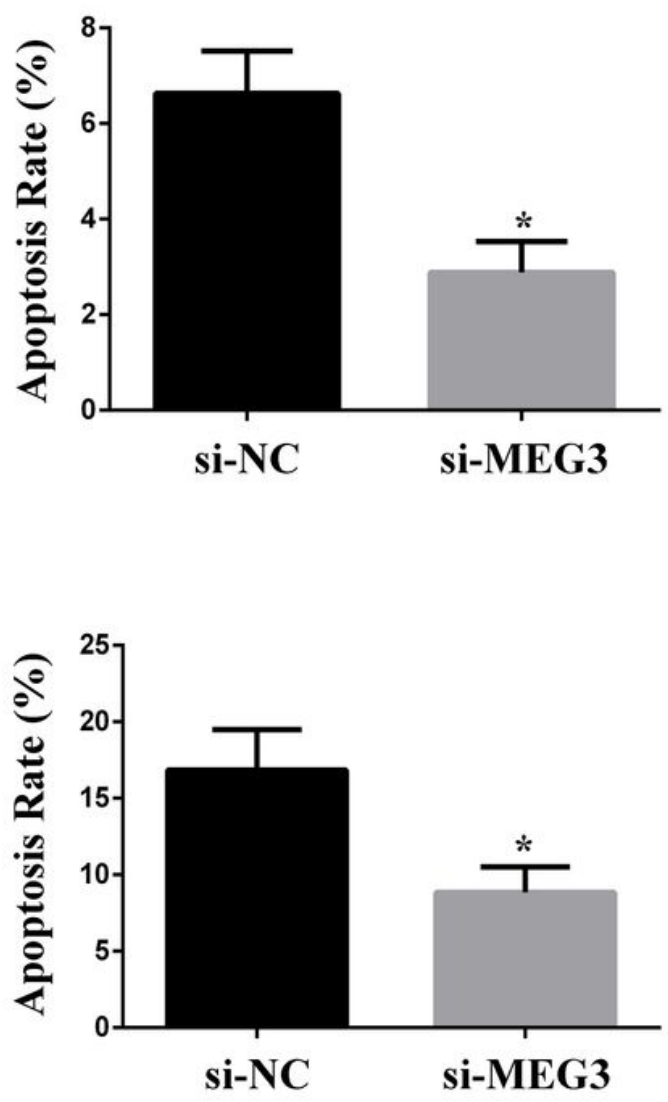

B

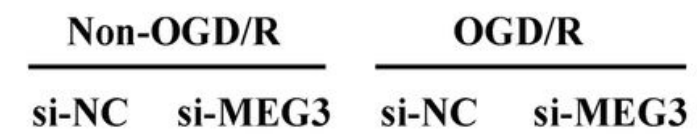

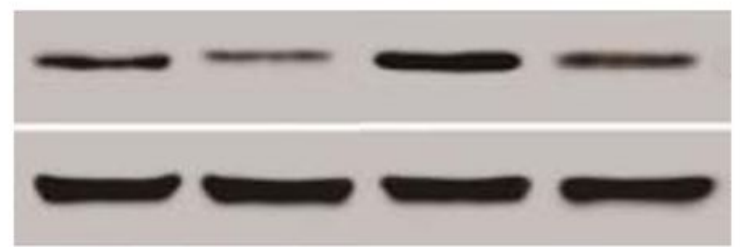

Figure 2

Apoptosis of HBMECs in response to various disturbances. (A) Apoptosis of HBMECs in response to various disturbances assessed by flow cytometry. (B). Expression level of Bax was down regulated by siMEG3, while OGD/R increased its expression level. ${ }^{*} \mathrm{P}<0.05$. 

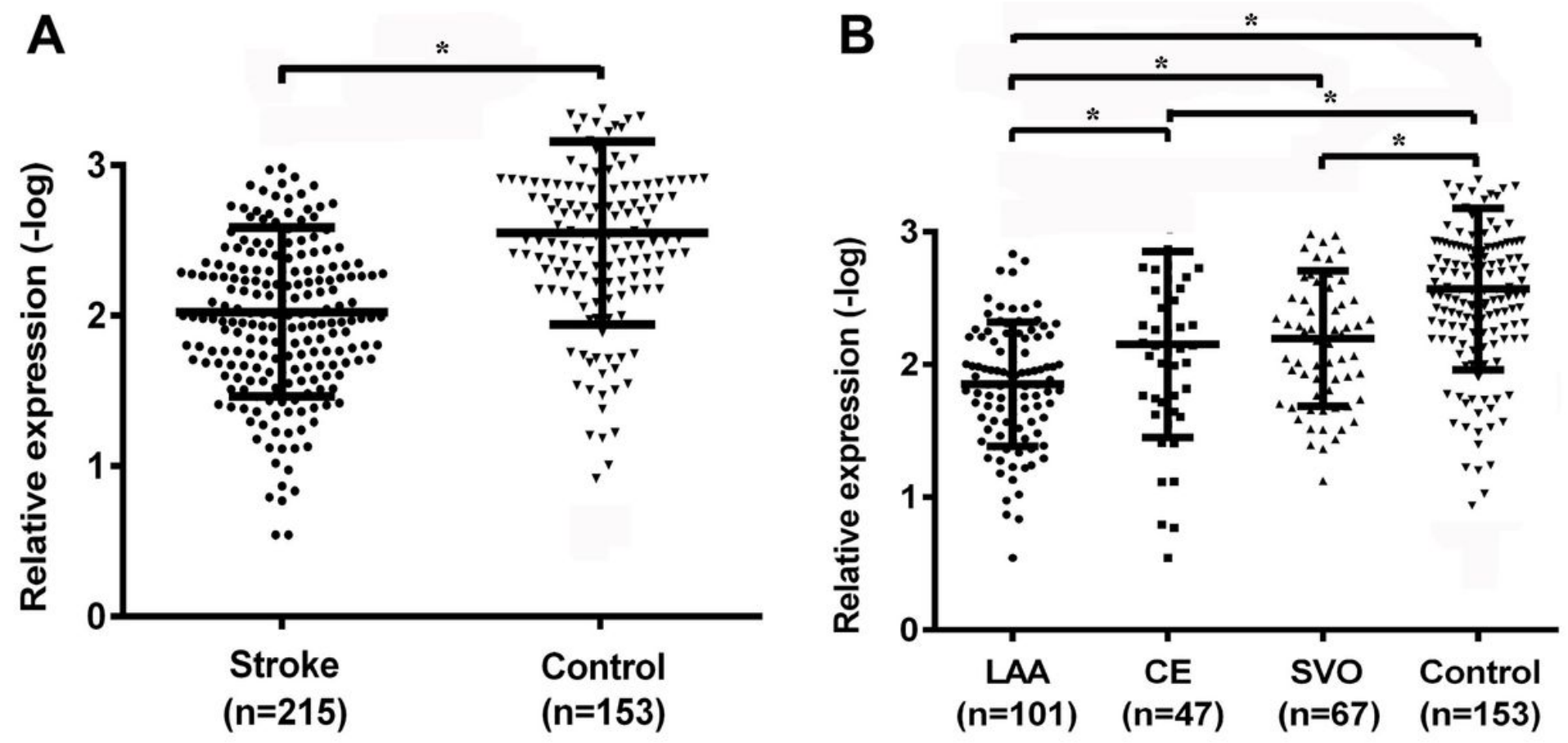

Figure 3

The MEG3 expression in peripheral blood leukocytes among subgroups. (A). MEG3 expression in ischemic stroke (IS) was significantly higher than that in the controls. (B). MEG3 expression in large-artery atherosclerosis (LAA) was significantly higher than that in cardioembolism (CE), small vessel occlusion (SVO) and the controls, and MEG3 expression in CE and SVO was significantly higher than that in the controls. ${ }^{*} \mathrm{P}<0.05$. 
A

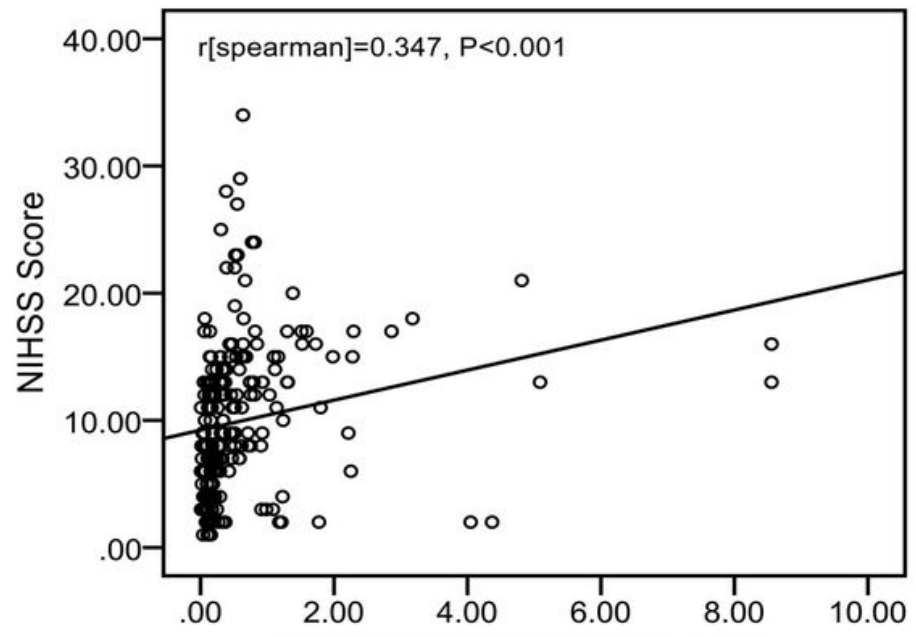

Expression level of MEG3

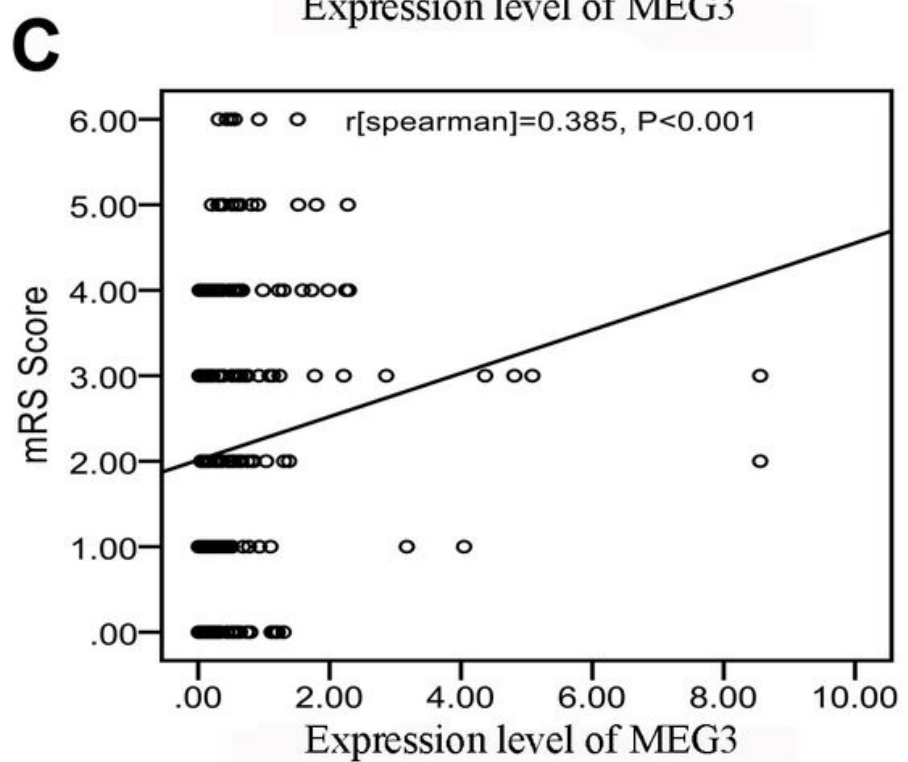

B

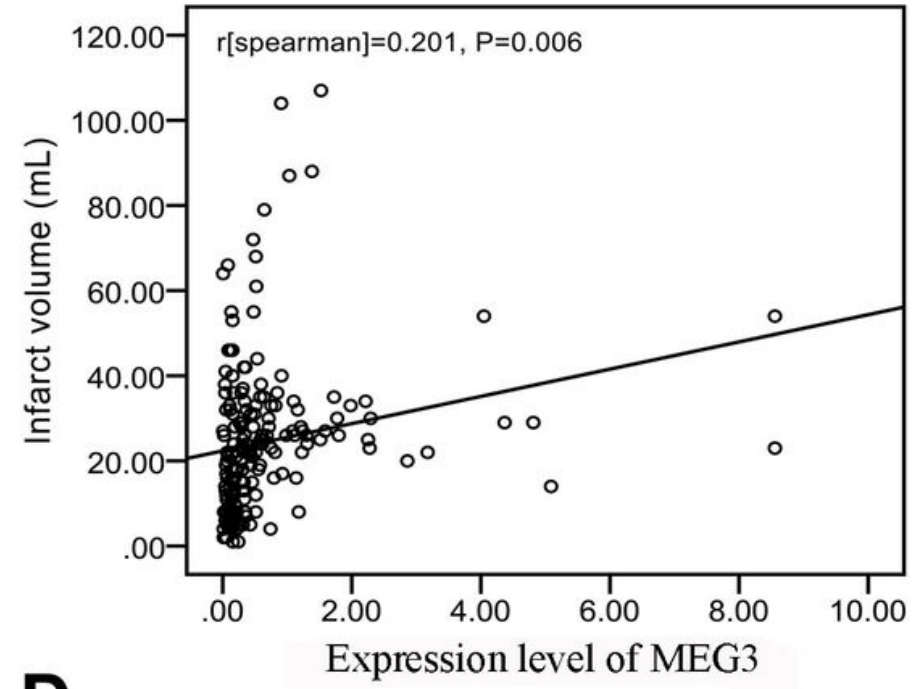

D

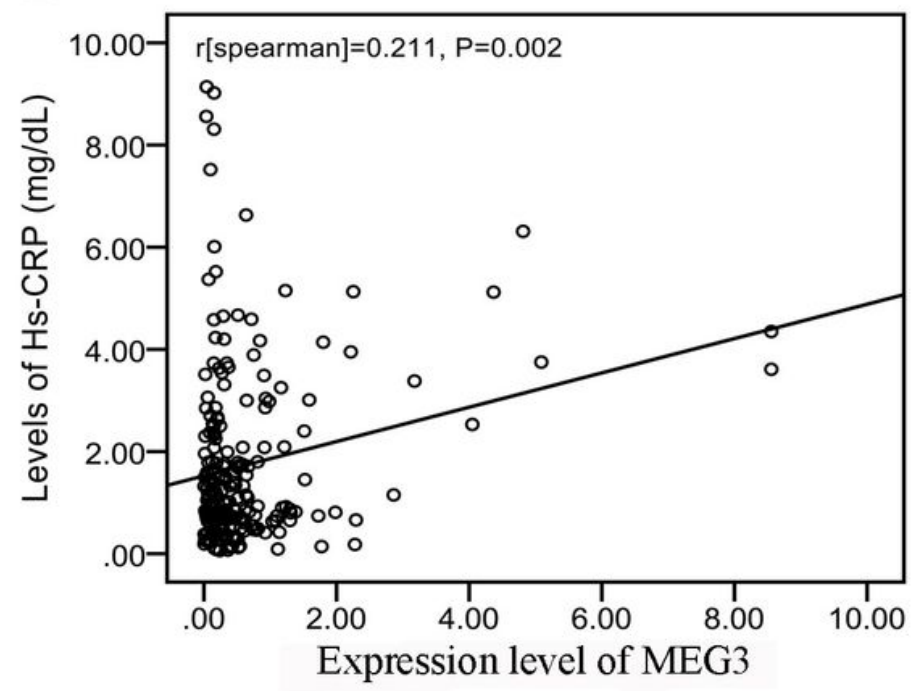

\section{Figure 4}

Correlation between MEG3 level and other predictors. (A) Correlation between MEG3 level and the National Institutes of Health Stroke Scale (NIHSS) score. (B) Correlation between MEG3 level and infract volume. (C) Correlation between MEG3 level and the modified Rankin Scale (mRS) score. (D) Correlation between MEG3 level and the level of Hs-CRP. 
A
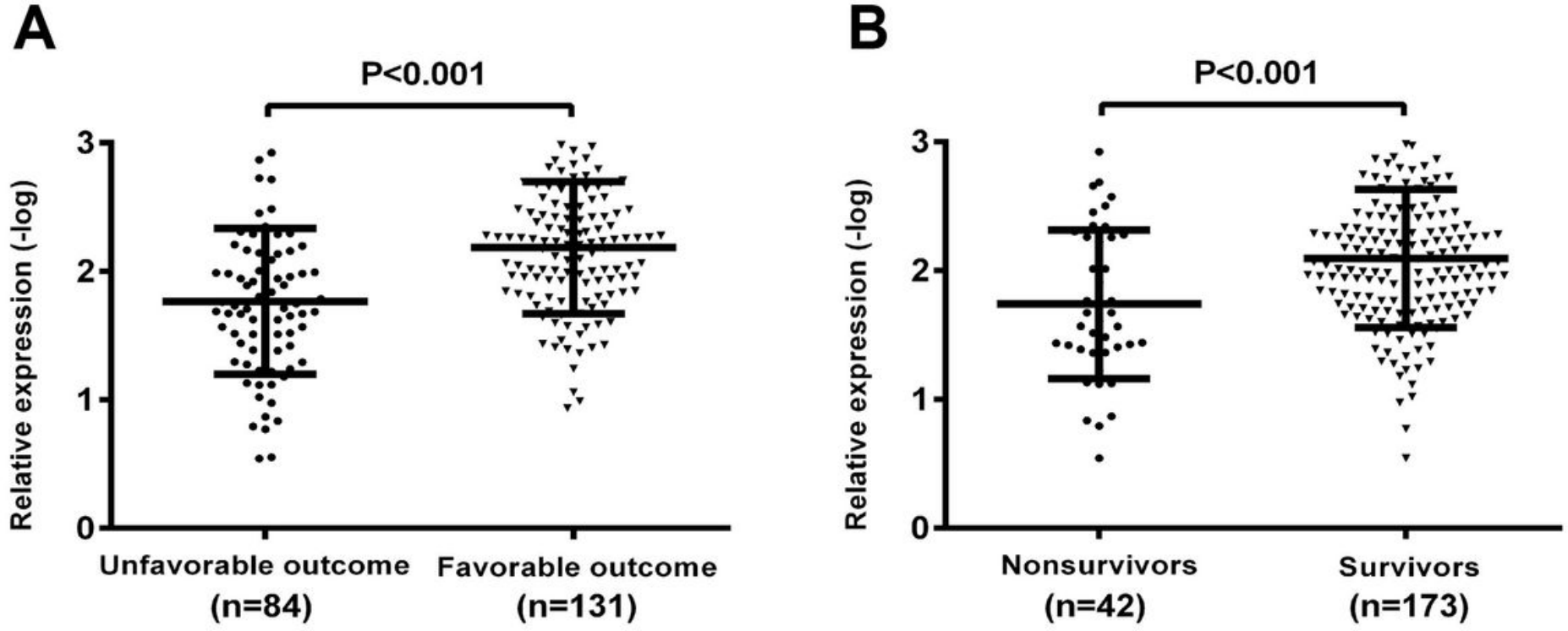

Figure 5

The MEG3 expression in peripheral blood leukocytes among subgroups. (A). MEG3 level in unfavorable outcome patients was significantly higher than that in the favorable outcome. (B). MEG3 level in nonsurvivors was significantly higher than that in survivors.

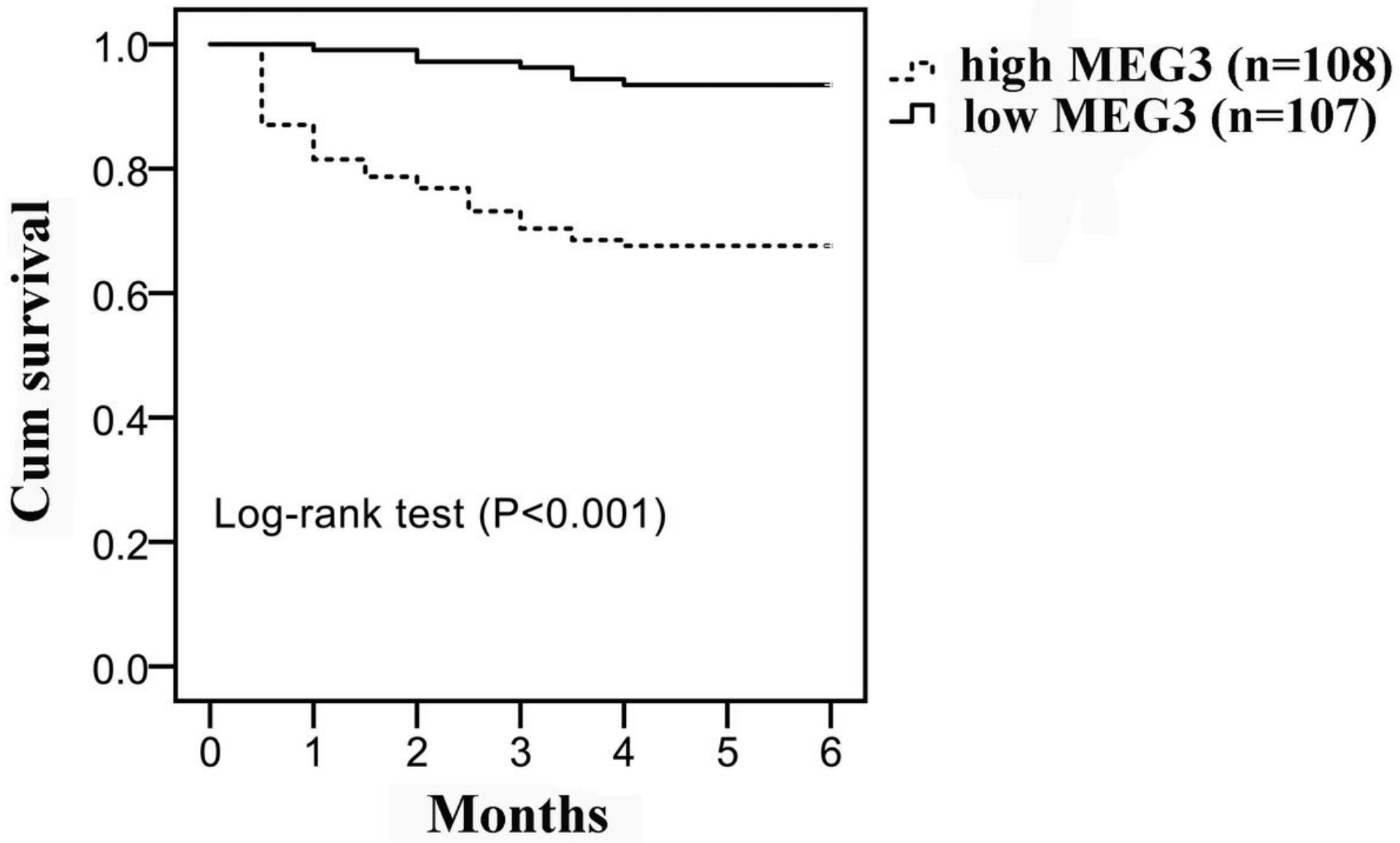

Figure 6 
Kaplan-Meier survival curves for MEG3. Patients in the high MEG3 group had a higher risk of death compared with low MEG3 group. Data analyzed using log-rank test.

\section{Supplementary Files}

This is a list of supplementary files associated with this preprint. Click to download.

- NC3RsARRIVEGuidelinesChecklistfillable.pdf 